\title{
Homogeneous Precipitation Synthesis and Magnetic Properties of Cobalt Ferrite Nanoparticles
}

\author{
Zhigang Liu, ${ }^{1,2}$ Xiaodong Li, ${ }^{1}$ Yonghua Leng, ${ }^{3}$ Jingbao Lian,, ${ }^{1}$ Shaohong Liu, ${ }^{1}$ Zhimeng Xiu, ${ }^{1}$ \\ Di Huo, ${ }^{1}$ Ji-Guang Li, ${ }^{1,3}$ and Xudong Sun ${ }^{1}$ \\ ${ }^{1}$ Key Laboratory for Anisotropy and Texture of Materials (Ministry of Education), School of Materials and Metallurgy, \\ Northeastern University, Shenyang 100004, China \\ ${ }^{2}$ Hebei Province Key Laboratory of Inorganic Nonmetallic Materials, College of Materials Science and Engineering, \\ Hebei Polytechnic University, Tangshan 063009, China \\ ${ }^{3}$ Nano Ceramics Center, National Institute for Materials Science, Namiki 1-1, Tsukuba, Ibaraki 305-0044, Japan
}

Correspondence should be addressed to Xudong Sun, xdsun@mail.neu.edu.cn

Received 27 October 2008; Accepted 27 December 2008

Recommended by Zhili Xiao

Magnetic nanoparticles (NPs) of cobalt ferrite have been synthesized via a homogeneous precipitation route using hexamethylenetetramine (HMT) as the precipitant. The particle size, crystal structure, and magnetic properties of the synthesized particles were investigated by X-ray diffraction, transmission electron microscopy, and vibrating sample magnetometer. The NPs are of cubic inverse spinel structure and nearly spherical shape. With the increase of oxidation time from 30 to 180 minutes in the reaction solution at $90^{\circ} \mathrm{C}$, the average particle size increases from $\sim 30 \mathrm{~nm}$ to $\sim 45 \mathrm{~nm}$. The as-synthesized NPs $\sim 30 \mathrm{~nm}$ in size show higher $\mathrm{M}_{\mathrm{s}}(61.5 \mathrm{emu} / \mathrm{g})$ and moderate $\mathrm{Hc}(945 \mathrm{Oe})$ and $\mathrm{M}_{\mathrm{r}} / \mathrm{M}_{\mathrm{s}}(0.45)$ value compared with the materials synthesized by coprecipitation method using $\mathrm{NaOH}$ as precipitate at high $\mathrm{pH}$ value.

Copyright (c) 2008 Zhigang Liu et al. This is an open access article distributed under the Creative Commons Attribution License, which permits unrestricted use, distribution, and reproduction in any medium, provided the original work is properly cited.

\section{INTRODUCTION}

Cobalt ferrite nanoparticles (NPs) with an inverse spinel structure are promising materials for high-density recording applications because of their high magnetocrystalline anisotropy, high coercivity, moderate saturation magnetization, and high chemical and structural stability at higher temperatures [1-3]. Recently, cobalt ferrite NPs have attracted great interest in the biomedical field, especially in magnetic targeted drug delivery and in magnetic fluid hyperthermia (MFH), due to their larger magnetic anisotropy and larger magnetic moments than iron oxides [4]. Cobalt ferrite NPs have been synthesized using various methods, such as coprecipitation in an aqueous solution [1], hydrothermal synthesis [5], microemulsion method [6], solgel-like technique [7], and sonochemical reaction method [8]. Among them coprecipitation of iron and cobalt ions in alkaline medium (usually $\mathrm{NaOH}$ and $\mathrm{NH}_{3} \cdot \mathrm{H}_{2} \mathrm{O}$ ) has proved to be a convenient and inexpensive synthesis route suitable to synthesize large batches of materials with reasonable control of composition and particle size. However, the local heterogeneous distribution of precipitant and metal ions during the reaction may lead to the precipitation of NPs with a relatively broad size distribution. To reduce the polydispersity of particle size, several groups have used microemulsions or vesicles at room temperature. However, with these techniques the crystallinity of the product is poor and the amount of material produced is highly reduced [9]. In this paper, a new homogeneous precipitation route was developed to synthesize cobalt ferrite NPs using hexamethylenetetramine (HMT) as the precipitant. The magnetic properties of cobalt ferrite NPs as synthesized by this method were also investigated in this paper.

\section{EXPERIMENTAL SECTION}

All the reagents used in this experiment, $\left(\mathrm{CH}_{2}\right)_{6} \mathrm{~N}_{4}(\mathrm{HMT})$, $\mathrm{FeCl}_{2} \cdot 4 \mathrm{H}_{2} \mathrm{O}$, and $\mathrm{Co}\left(\mathrm{NO}_{3}\right)_{2} \cdot 6 \mathrm{H}_{2} \mathrm{O}$, were of analytical grade and were used without any further purification. Required weights of $\mathrm{FeCl}_{2} \cdot 4 \mathrm{H}_{2} \mathrm{O}, \mathrm{Co}\left(\mathrm{NO}_{3}\right)_{2} \cdot 6 \mathrm{H}_{2} \mathrm{O}$, and HMT were dissolved into distilled water to achieve a mixed aqueous 
TABLE 1: Summaries of some properties of $\mathrm{CoFe}_{2} \mathrm{O}_{4}$ NPs derived from XRD, TEM, and magnetic measurement.

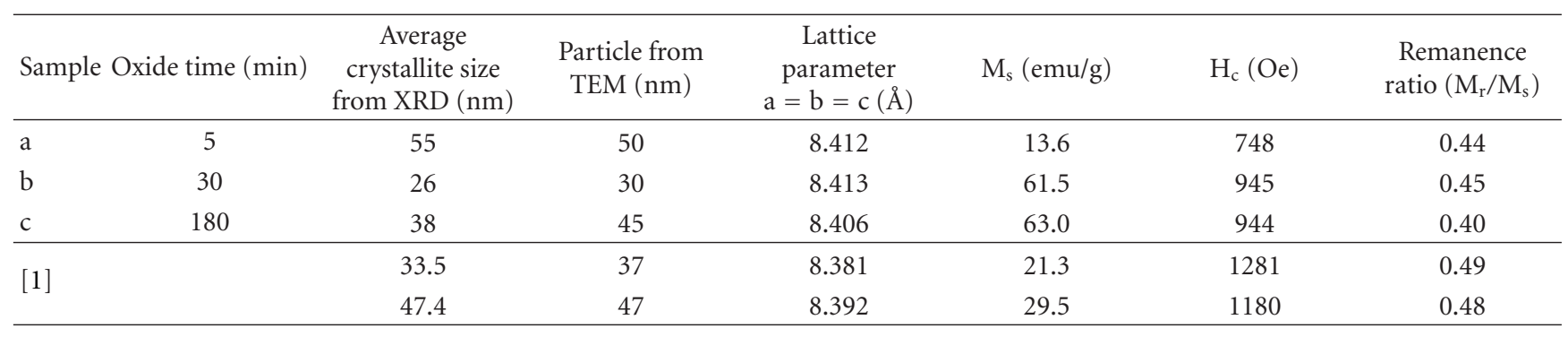

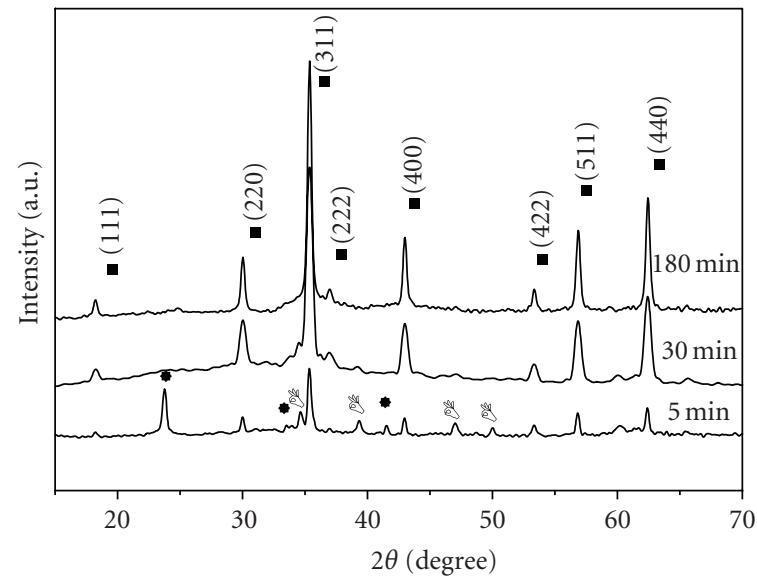

- $\mathrm{CoFe}_{2} \mathrm{O}_{4}$

- $\mathrm{Fe}(\mathrm{OH})_{3}$

$\mathrm{FeO}(\mathrm{OH})$

FIGURE 1: XRD patterns of precursory precipitate oxidized for 5, 30, and 180 minutes.

solution. The mixed aqueous solution was bubbled for 30 minutes by argon under magnetic agitating at room temperature to reduce the dissolved oxygen. The concentration of $\mathrm{Me}^{2+}\left(\mathrm{Me}^{2+}\right.$ represents $\mathrm{Fe}^{2+}$ and $\left.\mathrm{Co}^{2+}\right)$ was kept at $0.0125 \mathrm{M}$, with mole ratio of $\mathrm{Fe}^{2+} / \mathrm{Co}^{2+}$ at 2 and $\mathrm{HMT} / \mathrm{Me}^{2+}$ at 10 in the mixed solution. The temperature of the mixed solution was elevated to $80 \pm 2^{\circ} \mathrm{C}$ by silicon oil bath within 30 minutes and kept at the temperature for 1 hour to finish the coprecipitation reaction. Sage green alloy precipitate (partially oxidized $\mathrm{Fe}(\mathrm{OH})_{2}$ by $\mathrm{NO}_{3}{ }^{-}$or residual dissolved $\mathrm{O}_{2}$ in solution) defined as precursory precipitate was obtained. Thereafter, the reaction temperature was elevated to $90^{\circ} \mathrm{C}$ as quickly as possible and argon protection was then removed. Without the argon protection the reaction solution contacted with the air directly and concentration of dissolved $\mathrm{O}_{2}$ in solution increased. The obtained precursory precipitate then oxidized rapidly into black cobalt ferrite $\mathrm{NPs}_{3}$ by $\mathrm{NO}_{3}{ }^{-}$in solution and dissolved $\mathrm{O}_{2}$ from atmosphere. The precursory precipitate was oxidized for 5, 30, and 180 minutes, respectively, and then withdrawn from the reaction vessel. The products were separated by decanting clear solution using a $2.0 \mathrm{~T}$ permanent magnet to retain the NPs. All the samples were then washed with distilled water for 4 times and ethanol for 2 times, and then dried at $80^{\circ} \mathrm{C}$ in air for 10 hours. Production yield of this method with the oxidation time of 30 minutes was measured for three times and an average of $98 \mathrm{wt} \%$ was achieved. The small percentage of loss was induced during the decanting stages.

Phase identification was performed by X-ray diffractometry (XRD, Mode PW3040/60, Philips, Eindhoven, The Netherlands). Lattice constants were calculated based on the XRD patterns using software package of X'Pert HighScore Plus version 2.0 (PANanalytical B. V. Almelo, The Netherlands). The morphology and the particle sizes of the samples were observed using transmission electron microscopy (TEM, Model, 200CX, JEOL, Tokyo, Japan). The magnetization loops for the samples were measured using a vibrating sample magnetometer (VSM, JDM-13). The actual stoichiometric composition of the obtained powders was determined by a Shimadzu ICPS-75000 inductively coupled plasma atomic emission spectrometer (ICP-AES).

\section{RESULTS AND DISCUSSION}

At elevated temperatures, HMT releases precipitating ligand $\left(\mathrm{OH}^{-}\right)$homogeneously into the reaction system by decomposing into formaldehyde and ammonia species [10]. Because of the similar solubility of $\mathrm{Fe}(\mathrm{OH})_{2}$ and $\mathrm{Co}(\mathrm{OH})_{2}$, it is possible for iron $\left(\mathrm{Fe}^{2+}\right)$ and cobalt $\left(\mathrm{Co}^{2+}\right)$ ions to coprecipitate by reacting with precipitating ligand $\left(\mathrm{OH}^{-}\right)$to form hydroxide alloy precipitate under argon protection. Cobalt ferrite NPs are obtained through the oxidation of hydroxide alloy precipitate in air atmosphere. The cubic $\mathrm{CoFe}_{2} \mathrm{O}_{4}$ (JCPDS PDF no. 077-0426), orthorhombic Fe(OH) 3 (JCPDS PDF no. 046-1436), and tetragonal $\mathrm{FeO}(\mathrm{OH})$ (JCPDS PDF no. 075-1594) phases were detected by XRD for the precipitate oxidized for 5 minutes (Figure 1). After oxidation for 30 minutes, pure cubic $\mathrm{CoFe}_{2} \mathrm{O}_{4}$ was obtained, as confirmed by the XRD pattern, in which all the peaks were indexed to the cubic $\mathrm{CoFe}_{2} \mathrm{O}_{4}$ (JCPDS PDF no. 077-0426). In addition, the elemental analyses of these NPs using ICP-AES showed that the molar ratio of $\mathrm{Fe}^{3+} / \mathrm{Co}^{2+}$ is close to 2.0. The peaks of XRD patterns became sharper as the oxidation time increased from 30 to 180 minutes, indicating that grain size of the $\mathrm{CoFe}_{2} \mathrm{O}_{4} \mathrm{NPs}$ increases with the increase of the oxidation time. The average crystallite size was estimated from the full width at half maximum (FWHM) values of diffraction peaks (440) and (311) using the Scherrer formula, and the results are summarized in Table 1 . The crystalline sizes are 26 and $38 \mathrm{~nm}$ for the oxidation time of 30 and 180 minutes, 


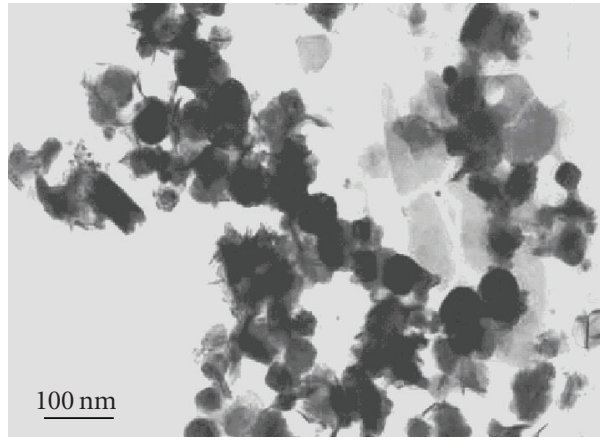

(a)

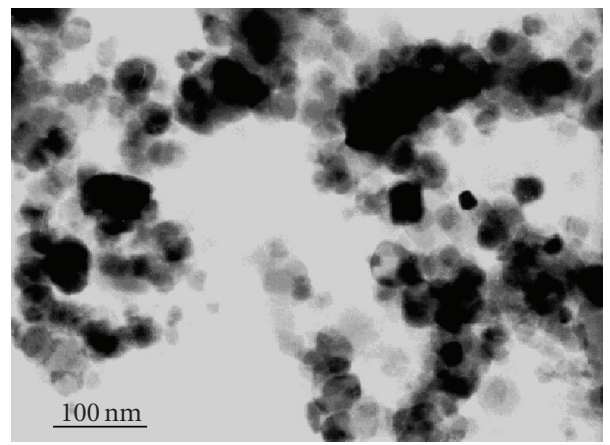

(b)

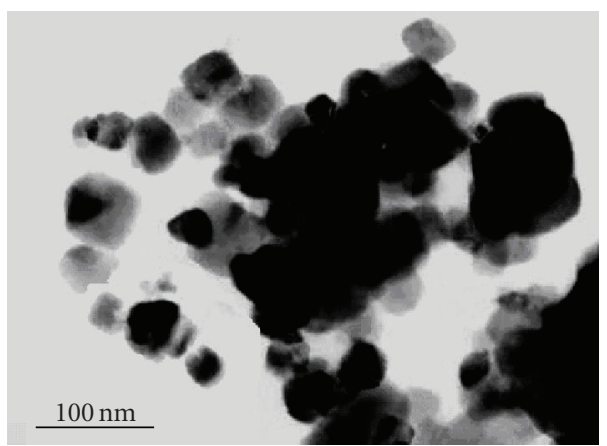

(c)

FIGURE 2: TEM photographs of the products oxidized for various times, (a) for 5 minutes, (b) for 30 minutes, and (c) for 180 minutes.

respectively. Lattice parameter (a) was calculated and listed in Table 1. The constants are close to the value reported in the literature (JCPDS PDF no. 077-0426, $\mathrm{a}=8.4000 \AA$ ).

Figure 2 shows the TEM photographs of the products oxidized for various times. Precursor oxidized for 5 minutes is mainly composed of nearly spherical $\mathrm{CoFe}_{2} \mathrm{O}_{4} \mathrm{NPs}$ $\sim 50 \mathrm{~nm}$ in size, with tiny acicular particles around nearly spherical $\mathrm{CoFe}_{2} \mathrm{O}_{4}$ NPs. The platelet and tiny acicular particles in Figure 2(a) were ascribed to the residual iron hydroxide phase. After oxidation for 30 minutes, the acicular and platelet particles disappeared and only nearly spherical $\mathrm{CoFe}_{2} \mathrm{O}_{4} \mathrm{NPs} \sim 30 \mathrm{~nm}$ in size were observed, as shown in Figure 2(b). This result is in agreement with the analysis of XRD patterns. These $\mathrm{CoFe}_{2} \mathrm{O}_{4}$ NPs show some extent of aggregation owing to the magnetic attraction. Quasispherical

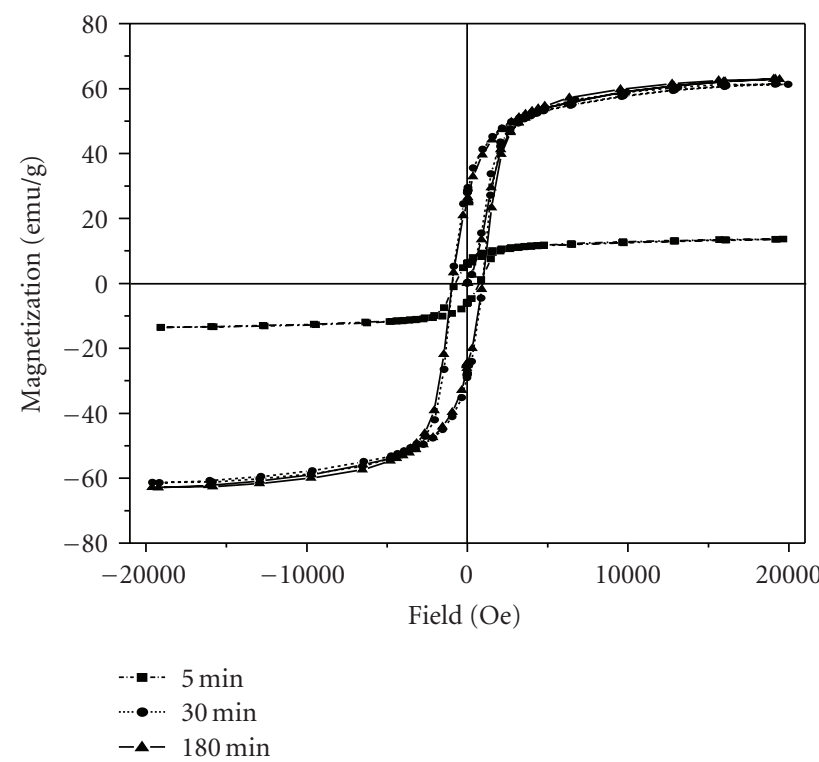

FIGURE 3: Hysteresis loops of the products oxidized for 5, 30, and 180 minutes at room temperature.

instead of spherical $\mathrm{CoFe}_{2} \mathrm{O}_{4}$ NPs were formed likely owing to the low solution $\mathrm{pH}$ value (about 7.5), which slowed down the nucleation and growth rate of spinel phase [11]. It is well known that faster growth rate usually favors the formation of spherical particles as a result of less selective crystallographic growth direction. Further, increase the oxidation time to 180 minutes, the particle size of $\mathrm{CoFe}_{2} \mathrm{O}_{4}$ NPs increased to $\sim 45 \mathrm{~nm}$ and more cubic particles appeared (Figure 2(c)). The average crystallite sizes from XRD are in reasonable agreement with those from direct observation from TEM images.

Figure 3 shows the hysteresis loops at room temperature for the samples oxidized for 5, 30, and 180 minutes. All the samples exhibit hysteresis loops typical of magnetic behaviors, indicating the presence of ordered magnetic structure in the spinel system. The saturation magnetization $\left(M_{s}\right)$, coercivity $\left(H_{c}\right)$, and remanence ratio $\left(M_{r} / M_{s}\right)$ are listed in Table 1. The $M_{s}$ value of the sample oxidized for 5 minutes is $13.6 \mathrm{emu} / \mathrm{g}$, significantly lower than that of the sample oxidized for 30 minutes $(61.5 \mathrm{emu} / \mathrm{g})$. This result is reasonable considering the existence of residual hydroxide phase. The $\mathrm{M}_{\mathrm{s}}$ value for the sample oxidized for 30 minutes is slight lower than those reported for the bulk samples $(>70 \mathrm{emu} / \mathrm{g})$. This can be attributed to the surface effects aroused by the distortion of the magnetic moments at the surface of nanocrystallite [1]. Although particle size is increased as the oxidation time increases from 30 to 180 minutes, the magnetic properties of $M_{s}, H_{c}$, and $M_{r} / M_{s}$ show little variations. This result is reasonable considering the particle size increment is small and particles are rather big so that the influence of the surface on the magnetic properties is low compared to the contribution of the volume of the particles. Compared with the corresponding counterpart materials synthesized by coprecipitation method using $\mathrm{NaOH}$ as precipitate at high $\mathrm{pH}$ value, the values of 
$\mathrm{M}_{\mathrm{s}}$ are higher and $\mathrm{H}_{\mathrm{c}}$ and $\mathrm{M}_{\mathrm{r}} / \mathrm{M}_{\mathrm{s}}$ are moderate, as shown in Table 1.

\section{CONCLUSION}

Cobalt ferrite NPs about $30 \mathrm{~nm}$ in size were synthesized by a new homogeneous precipitation routes using HMT as precipitant at low $\mathrm{pH}$ environment. This method for the synthesis of Cobalt ferrite NPs is simple, low in reaction temperature, high yielding, and inexpensive, without using expensive equipment and reactants.

\section{ACKNOWLEDGMENTS}

This work was supported by Program for New Century Excellent Talents in University (NCET-25-0290), the National Science Fund for Distinguished Young Scholars (50425413), and the National Natural Science Foundation of China (50672014).

\section{REFERENCES}

[1] Y. Qu, H. Yang, N. Yang, Y. Fan, H. Zhu, and G. Zou, "The effect of reaction temperature on the particle size, structure and magnetic properties of coprecipitated $\mathrm{CoFe}_{2} \mathrm{O}_{4}$ nanoparticles," Materials Letters, vol. 60, no. 29-30, pp. 35483552, 2006.

[2] M. H. Sousa, F. A. Tourinho, J. Depeyrot, G. J. da Silva, and M. C. F. L. Lara, "New electric double-layered magnetic fluids based on copper, nickel, and zinc ferrite nanostructures," The Journal of Physical Chemistry B, vol. 105, no. 6, pp. 1168-1175, 2001.

[3] Z. Chen and L. Gao, "Synthesis and magnetic properties of $\mathrm{CoFe}_{2} \mathrm{O}_{4}$ nanoparticles by using PEG as surfactant additive," Materials Science and Engineering: B, vol. 141, no. 1-2, pp. 8286, 2007.

[4] G. Baldi, D. Bonacchi, M. C. Franchini, et al., "Synthesis and coating of cobalt ferrite nanoparticles: a first step toward the obtainment of new magnetic nanocarriers," Langmuir, vol. 23, no. 7, pp. 4026-4028, 2007.

[5] A. Cabañas and M. Poliakoff, "The continuous hydrothermal synthesis of nano-particulate ferrites in near critical and supercritical water," Journal of Materials Chemistry, vol. 11, no. 5, pp. 1408-1416, 2001.

[6] Y. Ahn, E. J. Choi, S. Kim, and H. N. Ok, "Magnetization and Mössbauer study of cobalt ferrite particles from nanophase cobalt iron carbonate," Materials Letters, vol. 50, no. 1, pp. 4752, 2001.

[7] J.-G. Lee, J. Y. Park, and C. S. Kim, "Growth of ultra-fine cobalt ferrite particles by a sol-gel method and their magnetic properties," Journal of Materials Science, vol. 33, no. 15, pp. 3965-3968, 1998.

[8] K. V. P. M. Shafi, A. Gedanken, R. Prozorov, and J. Balogh, "Sonochemical preparation and size-dependent properties of nanostructured $\mathrm{CoFe}_{2} \mathrm{O}_{4}$ particles," Chemistry of Materials, vol. 10, no. 11, pp. 3445-3450, 1998.

[9] A.-T. Ngo and M.-P. Pileni, "Nanoparticles of cobalt ferrite: influence of the applied field on the organization of the nanocrystals on a substrate and on their magnetic properties," Advanced Materials, vol. 12, no. 4, pp. 276-279, 2000.
[10] T. C. Rojas and M. Ocaña, "Uniform nanoparticles of $\operatorname{Pr}(\mathrm{III}) /$ Ceria solid solutions prepared by homogeneous precipitation," Scripta Materialia, vol. 46, no. 9, pp. 655-660, 2002.

[11] R. T. Olsson, G. Salazar-Alvarez, M. S. Hedenqvist, U. W. Gedde, F. Lindberg, and S. J. Savage, "Controlled synthesis of near-stoichiometric cobalt ferrite nanoparticles," Chemistry of Materials, vol. 17, no. 20, pp. 5109-5118, 2005. 

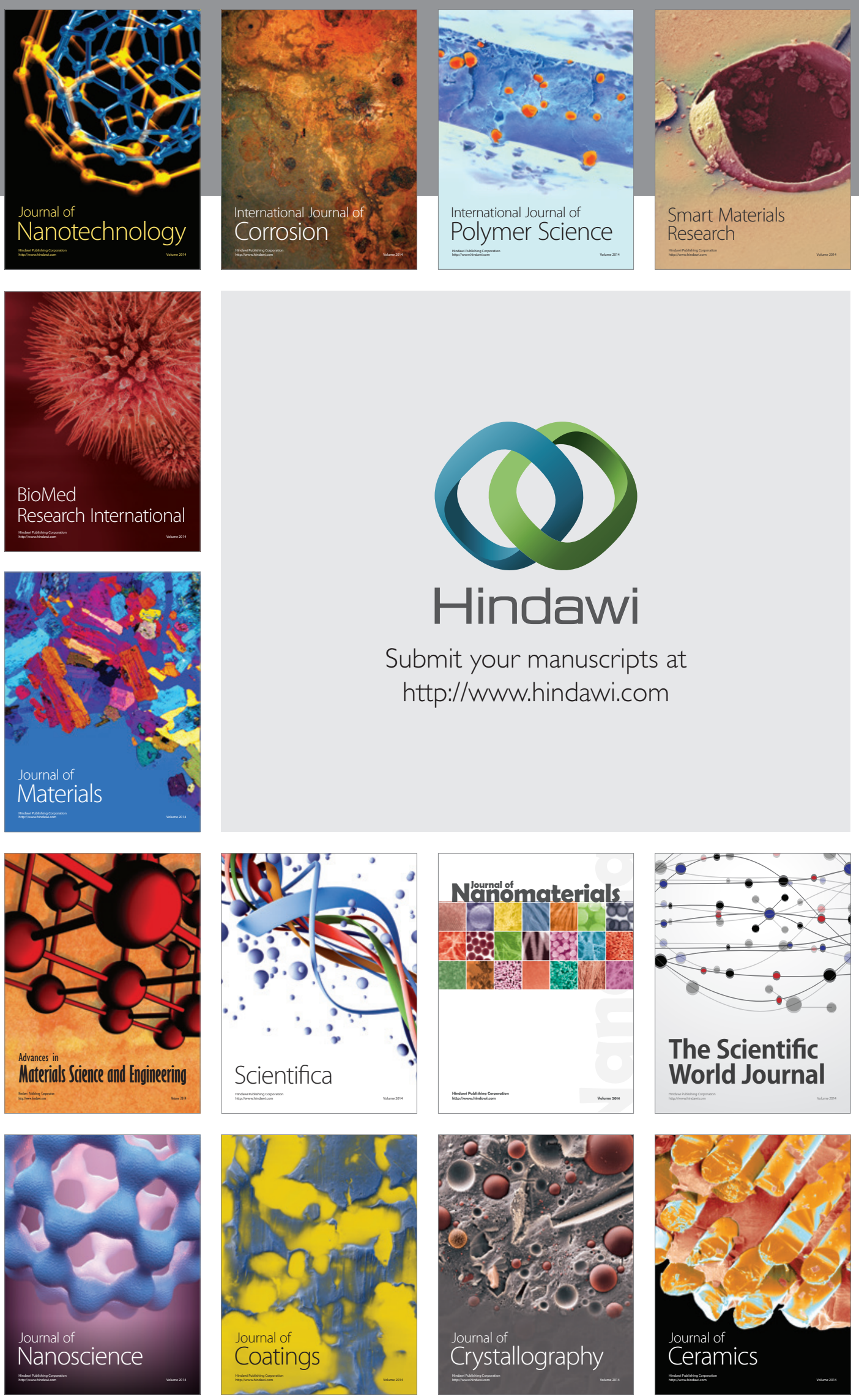

The Scientific World Journal

Submit your manuscripts at

http://www.hindawi.com

\section{World Journal}

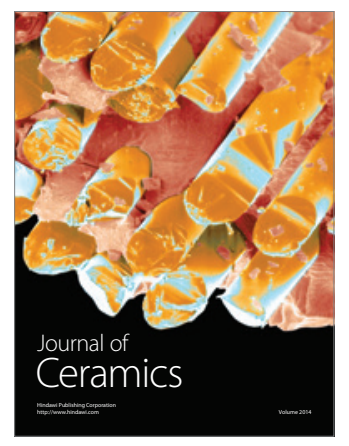

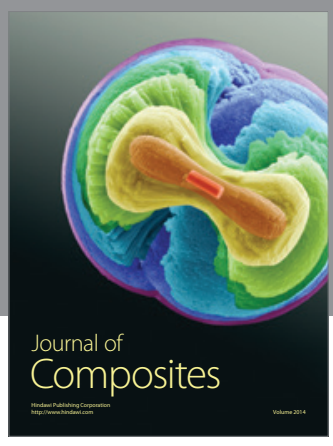
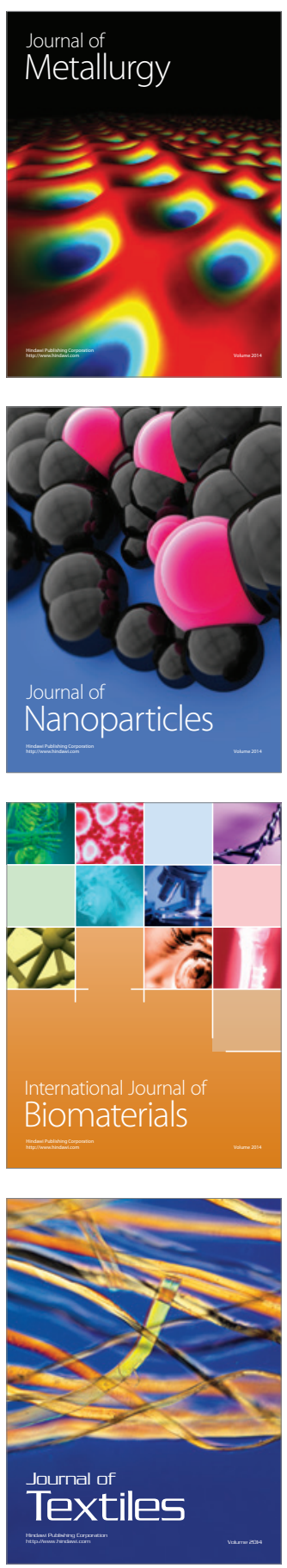